\title{
Surface Enhanced Raman Spectroscopy of Unilamellar Liposomes Loaded with Silver Nanoparticles
}

\author{
Pelin Toren ${ }^{1}$, Turgay Tekinay², 3 , Sencer Ayas ${ }^{1}$, Aykutlu Dana ${ }^{1}$, and IIknur Tunc ${ }^{4, *}$ \\ ${ }^{1}$ UNAM-National Nanotechnology Research Center and Institute of Materials Science and Nanotechnology, \\ Bilkent University, 06800 Ankara, Turkey \\ ${ }^{2}$ Gazi University, Life Sciences Application and Research Center 06830 Ankara, Turkey \\ ${ }^{3}$ Gazi University, Polatı Science and Literature Faculty, 06900 Ankara Turkey \\ ${ }^{4}$ University of Turkish Aeronautical Association, Turkkusu Campus, 06790 Ankara, Turkey
}

\begin{abstract}
Imaging organic molecules using surface-enhanced Raman spectroscopy (SERS) has drawn attention due to its non-invasive nature and label-free approach. The SERS approach can be used in tracking organic molecules and monitoring unique Raman spectra of the organic molecules bound to metal nanoparticles (NPs). We prepared unilamellar liposomes composed of 1,2-dipalmitoylsn-glycero-3-phosphocholine (DPPC) molecules with a diameter of around $100 \mathrm{~nm}$. Electrostatic binding of silver $(\mathrm{Ag}) \mathrm{NPs}$ on the surface of the unilamellar liposomes was achieved by the reduction of silver nitrate $\left(\mathrm{AgNO}_{3}\right)$ which produces SERS active silver colloidal particles on the unilamellar liposome surfaces. Highly enhanced electromagnetic fields localized around neighbouring Ag NPs provide hot-spot construction around the liposomes, due to the spatial distribution of SERS enhancement in the unilamellar liposomes. It was observed that the signals fluctuate on a second time scale, presumably due to conformational change of DPPC chain and local Brownian motion of liposomal spheres.
\end{abstract}

Keywords: Surface-Enhanced Raman Spectroscopy (SERS), Hot-Spots, Liposomes, Silver Nanoparticles, Liposomes, ULVs.

\section{INTRODUCTION}

Liposomal delivery systems are commonly utilized for the storage, transfer and controlled release of various therapeutic agents, either by direct attachment onto the lipid bilayer or by incorporation into the enclosed internal aqueous compartments. Such systems are highly biodegradableand biocompatible, mainly due to their structural similarity with the cell membrane and various cellular organelles with lipid bilayers. ${ }^{1-3}$

Intracellular studies showed that liposomes can be used in drug delivery. ${ }^{1-3}$ Besides being used as carriers for drug delivery, large liposome vesicles have been widely used to mimic biological membranes and to study phospholipid oxidization, protein reconstitution and protein-protein or protein-lipid interactions..$^{4-8}$

In order to develop the next generation of liposomes as drug carriers, properties such as controllable release and

\footnotetext{
*Author to whom correspondence should be addressed.
}

in vivo traceability of the liposome should be improved by functionalization of its constituents. Towards this goal, noble metal nanoparticles (NPs) such as gold (Au) and silver (Ag) NPs can be used as functional molecules based on their unique physical and chemical properties. ${ }^{9,10}$ Due to their surface plasmon resonance (SPR) in the visible region, $\mathrm{Au}$ and $\mathrm{Ag}$ NPs can strongly absorb light energy in the visible regionand convert the absorbed light energy into heat. ${ }^{11,12}$ These nanoparticles have been considered for several applications such asphotothermal therapy, in vivo imaging and photosensitive drug release. ${ }^{13,14}$ $\mathrm{Au} / \mathrm{Ag}$ NPs incorporated liposomes have received considerable attention as novel stimuli responsive and traceabledrug carriers. ${ }^{4,15}$ However, such photochemical features are only expressed by Au/AgNPs of sizes smaller than $100 \mathrm{~nm}$ in diameter and aggregation of the NPs may block synergetic properties and functions..$^{9,10}$

Instead of bulk analysis, single molecule characterization techniques need to be used for characterization of

$1533-4880 / 2017 / 17 / 8894 / 007$

doi:10.1166/jnn.2017.13880 
liposomes to ensure reproducible preparation of the vesicles. Compared to bulk analysis, the techniques focusing on the individual analysis of liposomes can bring out heterogeneity masked by ensemble averaging. Recent advances in such techniques for individual liposome characterization have been recently published. ${ }^{16}$ Among such tecniques raman spectroscopy when integrated with confocal microscopy allows to examine lipid vesicle composition at the single-liposome level.

Raman spectroscopy is an attractive method for the study of lipid-protein interactions due to its non-invasive and non-destructive properties. This technique, however, suffers from sensitivity issues, while surface-enhanced Raman spectroscopy (SERS) offers increased sensitivity for molecular detection without the need for any marker molecule. Raman signal enhancement mainly arises from the electromagnetic effect (EM). Particularly, when an analyte is adsorbed onto metal NPs, gaps and junctions created between interconnected NPs, generates so-called 'hot-spots,' as they intensify Raman signal drastically, resulting in an enhancement factor strong enough to allow single molecule detection. ${ }^{5,6}$

Several studies demonstrate the applicability of a liposome-metal NP system for SERS-mediated bioimaging. Tam et al. ${ }^{17}$ prepared gold nanoparticle-based SERS probes encapsulating Au nanoparticles with raman active phospoholipid in order increase stability and biocompatibility of Au nanoparticles with liposome structure. Liu et al. ${ }^{18}$ discussed the SERS-derived signal from crystal violet $(\mathrm{CV})$ reporter molecules encapsulated in antibodymodified liposome particles. Liposome-mediated enhancement of the sensitivity in immunoassay was demonstrated.

However, to the best of our knowledge SERS study on the label free detection of liposome has not yet been performed. So far liposomes have been used for assisting purposes. Label-free detection of single liposomes might be vital to understand the mechanism of intracellular uptake of liposome-bound drugs and/or metal nanoparticles for curing. Also, since liposomes mimic cell membrane and various cellular organelles with lipid bilayers because of structural similarity, carbonization of liposomes even under very low laser exposure is very promising result for applicability of phototermal therapy. SERS is the only advanced method that is available at present for single molecule detection and detailed structure analysis, and is ideal for the detection of single liposomes in biological sensing and disease diagnostic applications. ${ }^{19-21}$

In this study, unilamellar liposomes or vesicles (ULVs) were synthesized by lipid hydration method and silver nitrate $\left(\mathrm{AgNO}_{3}\right)$ was then reduced onto the surfaces of the ULVs in the presence of sodium borohydride $\left(\mathrm{NaBH}_{4}\right)$ to observe SERS effect in Ag NPs loaded ULVs. The local SERS response was mapped over time by detecting the Raman signals from Ag NPs loaded ULVs deposited on the metallic Ag substrate.

\section{EXPERIMENTAL DETAILS}

\subsection{Materials}

1,2-Dipalmitoyl-sn-glycero-3-phosphocholine (DPPC, Avanti Polar Lipids Inc., Alabaster, AL; MW:734.04) was used to syhthesize ULVs. In order to prepare multilamellar vesicles (MLVs), 4-(2-hydroxyethyl)piperazine-1-ethane sulfonic acid (HEPES, Sigma-Aldrich company, St. Louis, MO; MW:238.30) and chloroform $\left(\mathrm{CHCl}_{3}\right.$, Sigma-Aldrich company, St. Louis, MO; MW:119.38) were used. A mini extruder (Avanti Polar Lipids Inc., Birmingham, AL) was used with track-etch polycarbonate membranes (Whatman Nuclepore company, Clifton, NJ) with uniform pore size of $100 \mathrm{~nm}$ for preparation of unilamellar vesicles (ULVs) which are the typical liposomes used in this study. Silver nitrate $\left(\mathrm{AgNO}_{3}\right.$, Sigma-Aldrich company, St. Louis, MO; MW:169.87) and sodium borohydride $\left(\mathrm{NaBH}_{4}\right.$, SigmaAldrich company, St. Louis, MO; MW:37.83) were used to synthesize Ag NPs. $10 \%$ (w/v) Phosphotungstic acid solution (PTA, Sigma-Aldrich company, St. Louis, MO) was used for contrast transmission electron microscopy (CTEM) imaging purposes. Milli-Q grade (Millipore Corporation, Bedford, MA), ultra pure water with resistivity greater than $18.0 \mathrm{M} \Omega \mathrm{cm}$ was used in all preparations.

\subsection{Preparation of ULVs}

In order to form ULVs, $0.07 \mathrm{~g}$ DPPC was dissolved in chloroform in an erlenmeyer flask. Chloroform was then evaporated overnight at room temperature in a fume hood in order to form a thin film at the bottom of the flask. The dry lipid film was hydrated in $50 \mathrm{ml}, 20 \mathrm{mM}$ HEPES buffer in ultrapure water at $\mathrm{pH}$ 7.4. The solution was vortexed at $80{ }^{\circ} \mathrm{C}$ until the lipid film couldno longer be observedat the bottom of the flask and MLVs were formed. The solution which contained MLVs was sonicated for 30 minutes and subsequently extruded ten times through a polycarbonate membrane with a pore size of $100 \mathrm{~nm}$ to obtain ULVs at a final lipid concentration of $2 \mathrm{mM}$.

\subsection{Preparation of Ag NPs Loaded ULVs}

AgNPs were formed in the presence of the negatively charged ULVs by the reduction of $\mathrm{Ag}^{+}$ions around vesicle surfaces. A lipid solution $(2 \mathrm{mM})$ containing ULVs was prepared as described above and used as stock. $\mathrm{A} \mathrm{AgNO}_{3}$ $(0.1 \mathrm{mM})$ solution in ultrapure water was added dropwise to the pre-diluted lipid solution $(1 \mathrm{mM})$ in pure water under vigorous stirring at room temperature until the molar ratio of DPCC: $\mathrm{AgNO}_{3}$ reached 10:1. The mixture was then stirred for 30 mins at room temperature to let $\mathrm{Ag}^{+}$ions bind electrostatically to the negatively charged ULVs. $\mathrm{Ag}^{+}$ ions were then reduced by dropwise addition of $2 \mathrm{mM}$ aqueous $\mathrm{NaBH}_{4}$ solution in ultrapure water to the mixture. A visible color change from colorless to yellow, within several minutes after addition of $\mathrm{NaBH}_{4}$, indicates the formation of AgNPs around the ULVs. In addition, bare colloidal $\mathrm{Ag}$ was prepared by adding an excess of $\mathrm{NaBH}_{4}$ to 
a

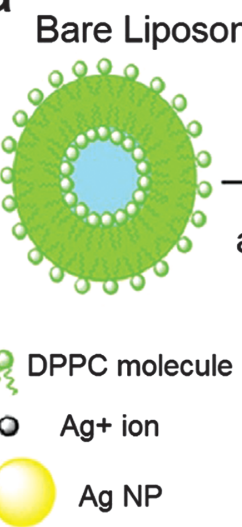

Ag NPs loaded Liposome
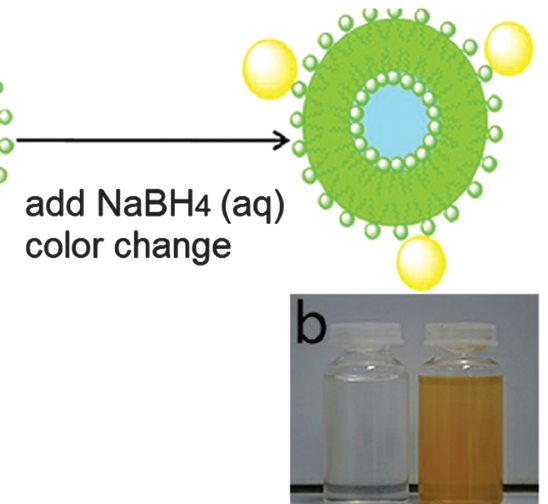

Figure 1. (a) An illustration of the experimental route of the preparation of Ag NPs loaded ULVs and (b) a photograph of solutions which contain bare ULVs and Ag NPs loaded ULVs shown on the left and right, respectively.

$0.1 \mathrm{mM} \mathrm{AgNO}_{3}$ solution under vigorous stirring at room temperature. Figure 1(a) shows the schematical representation of the procedure for the preparation of Ag NPs loaded onto ULVs. Figure 1(b) displays the drastic color change, from colorless to yellow, which indicates successful loading of the Ag NPs onto ULVs.

\subsection{Characterization of the Bare and Ag NPs Loaded ULVs}

Zeta Potential/Dynamic Light Scattering (DLS). Zeta potential and particle size distributions of the ULVs and Ag NPs loaded ULVs were determined by photon correlation spectroscopy using a Malvern Zetasizer Nano ZS90 DLS spectrometer system (Malvern Instruments, Worcestershire, UK). The samples were diluted in ultrapure water at 1:10 volume ratio for zeta size/potential analyses. 4 separate measurements were performed for each sample. Each measurement was averaged over 4 runs. Zeta size measurements were conducted with disposable sizing cuvettes at a scattering angle of $173^{\circ}$ at $25^{\circ} \mathrm{C}$. Zeta potential measurements were conducted with clear disposable zeta cells at $25^{\circ} \mathrm{C}$.

Contrast Transmission Electron Microscopy (CTEM). Morphology and size analyses of the ULVs and Ag NPs loaded ULVs were conductedwith a Tecnai $G^{2}$ Spirit Biotwin transmission electron microscope (FEI company, Hillsboro, OR) operated at an accelerating voltage of $80 \mathrm{kV}$ and with a line resulation of $0.34 \mathrm{~nm}$. Prior to measurement, samples were centrifuged at $1500 \mathrm{rpm}$ for 30 mins at $25^{\circ} \mathrm{C}$ and re-dispersed inultrapure water at a $1: 10$ volume ratio. Aliquots of UVLs and Ag NPs loaded UVLs were dropped on separate CTEM grids and the excess solutions were removed with filter papers. Negative staining was applied to all grids using a diluted PTA solution $(2 \% \mathrm{w} / \mathrm{v})$ in ultrapure water and excess of PTA solution was removed after 60 seconds using a filter paper. Then the CTEM grids were dried overnight before imaging.
$U V$-Visible (UV-Vis) Absorption Spectroscopy. UV-Vis absorption spectroscopy analyses of the ULVs and Ag NPs loaded ULVs were conducted with a NanoDrop 2000c spectrophotometer (Thermo Fisher Scientific company, Hampton, NH).

Surface Enhanced Raman Spectroscopy (SERS). SERS measurements were performed using a Alpha 300S scanning near-field optical microscope (SNOM) with Raman module (WITec company, Knoxville, TN). $100 \mu$ l of the solution containing Ag NPs loaded ULVs was dropped onto a polyvinyl pyrrolidone (PVP) deposited $70 \mathrm{~nm}$ metallic Ag film on silicon wafer (with a $1 \mathrm{~cm}^{2}$ surface area) and the SERS sample was dried at room temperature. A solid-state $532 \mathrm{~nm}$ wavelength laser (with excitation power $200 \mu \mathrm{W}$ ) was used to conduct SERS measurements. Raman signals were collected with confocal scanning mode through $100 \times$ magnification for SERS imaging.

\section{RESULTS AND DISCUSSION}

\subsection{The Bare and Ag NPs Loaded ULVs}

Figures 2( $\mathrm{a}$ and $\mathrm{b}$ ) show CTEM images of the bare ULVs and a single ULV loaded with Ag NPs, respectively. The presence of stable individual ULVs are clearly observed in Figure 2(a). In Figure 2(b), an individual Ag NP loaded ULV is displayed. The Ag NPs loaded onto an individual ULV are relatively smaller in size and can be clearly distinguished on the ULV. A small amount of the independent larger Ag NPs are also observed around the ULVs.

Figure 2(c) shows particle size $(\mathrm{nm})$ distribution of bare ULVs (red columns) and Ag NPs loaded ULVs (black columns) by number (\%). The average particle size and zeta potential of bare ULVs are $98.13 \mathrm{~nm}$ and $-4.48 \pm$ $2.63 \mathrm{mV}$, respectively. The average particle size and zeta potential of Ag NPs loaded ULVs are $151.80 \mathrm{~nm}$ and $+20.26 \pm 4.96 \mathrm{mV}$, respectively. The average particle sizes, measured with the DLS instrument, are in agreement with CTEM measurements. Loading Ag NPs onto the 

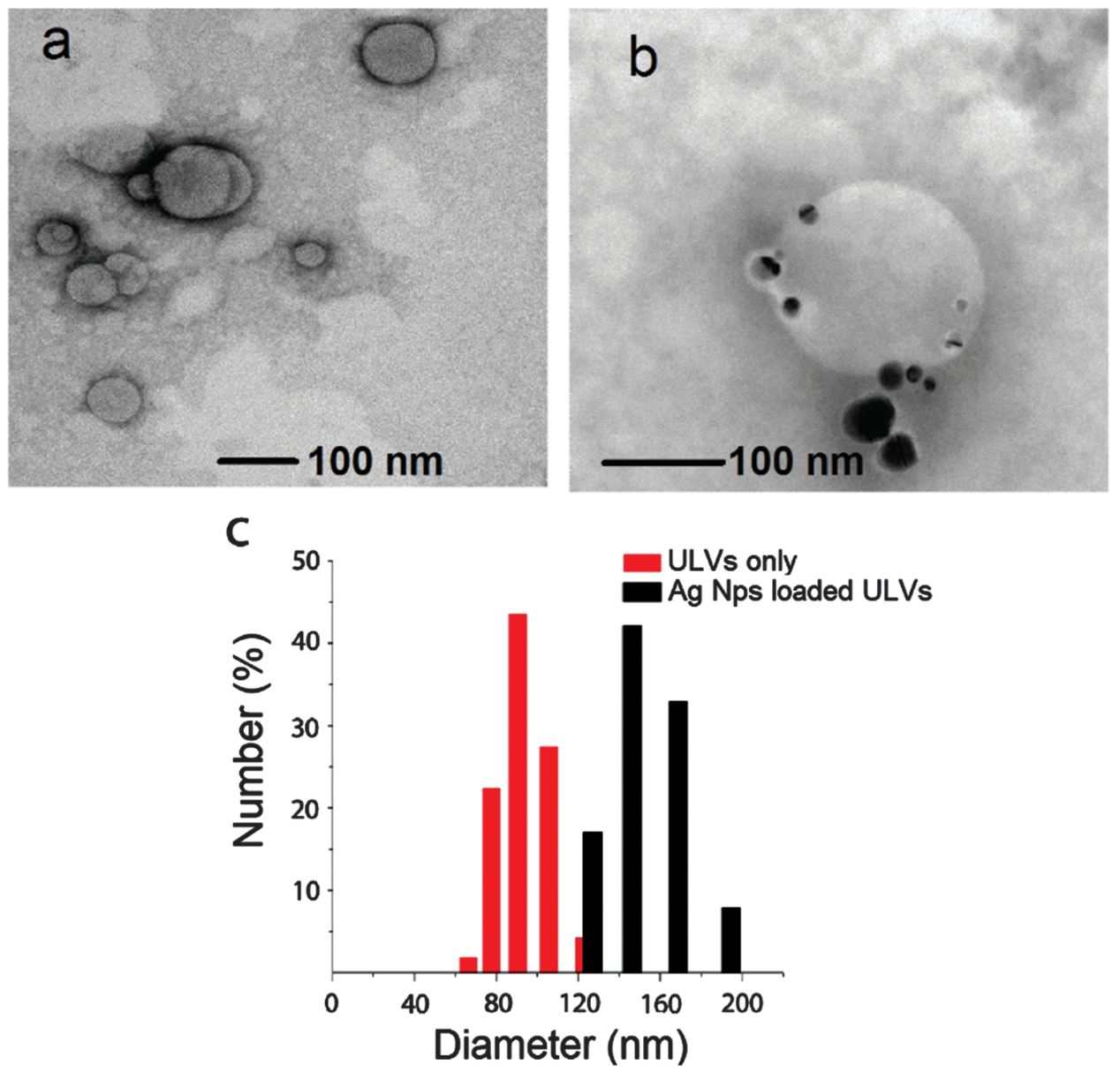

Figure 2. CTEM images of (a) bare unilamellar vesicles (ULVs) and (b) a ULV loaded with Ag NPs. (c) Size (nm) distribution of bare ULVs (red columns) and Ag NPs loaded ULVs (black columns) by number (\%).

preformed ULVs increase the average particle size of the ULVs as expected.

Figure 3 shows absorption spectra of the bare ULVs (red line), bare Ag NPs (black line) and AgNPs loaded ULVs (blue line). Bare Ag NPs have a characteristic Surface

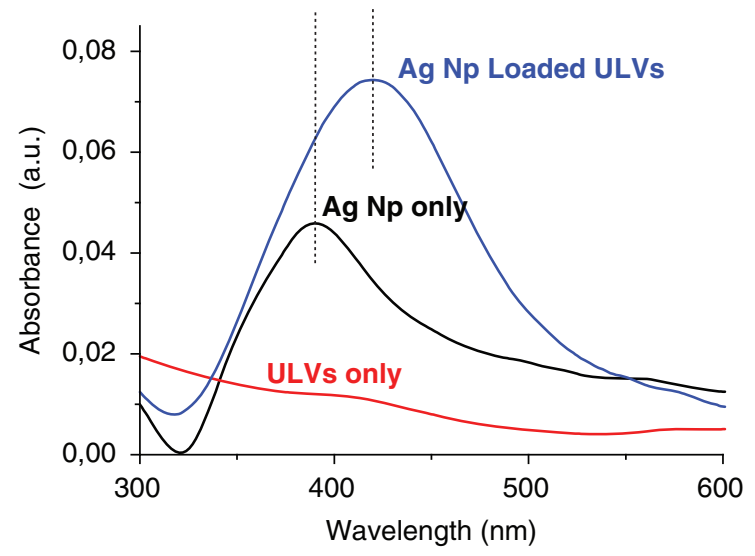

Figure 3. UV-Visible absorption spectra of aqeous solutions of bare ULVs (red line), bare Ag NPs (black line), and Ag NPs loaded ULVs (blue line).
Plasmon Resonance (SPR) band at around $400 \mathrm{~nm}$. The Ag NPs loaded on ULVs also display an SPR band located at around $420 \mathrm{~nm}$. The incorporation of ULVs with the Ag NPs leads to a red-shift at the SPR band of Ag NPs. This is due to the change of dielectricity around the Ag NPs upon deposition onto the ULVs. ${ }^{11}$

\subsection{Mapping the Local SERS Response}

Local SERS activity of the Ag NPs loaded ULVs were investigated. Time dependent SERS measurements were performed to observe the SERS activity of the Ag NPs loaded ULVs. Figure 4(a) shows the SERS image constructed by plotting the Raman band intensity over the scanned area. The constructed SERS enhancement map displays non-regular patterns of hot-spots generated at junctions between Ag NPs located randomly on the ULVs, suggesting that highly enhanced electromagnetic fields are localized around neighbouring Ag NPs. Figure 4(b) displays an illustration of how hot-spots are formed as a result of the SERS enhancement on DPPC molecules constituting the ULVs. The strongest SERS intensities are likely to be formed by extraversive functional groups of the DPPC 


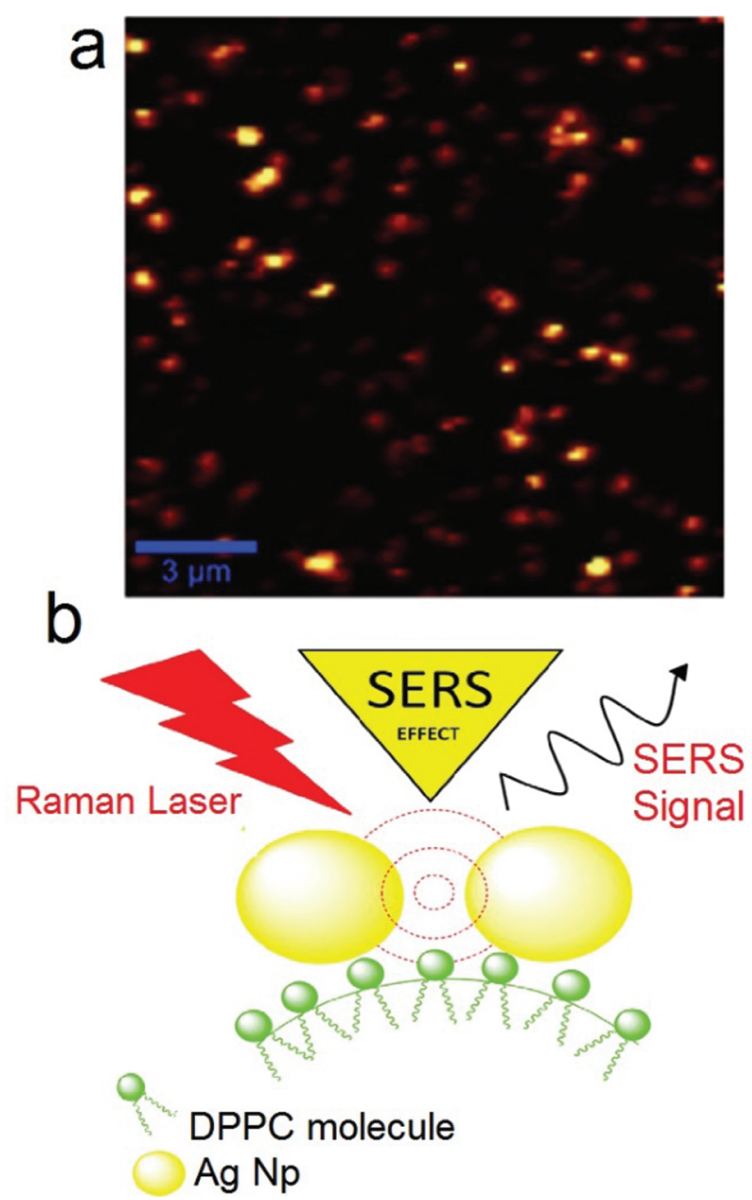

Figure 4. (a) Representative SERS image from the video sequence exhibits rapid and high density blinking spots. (b) An illustration of forming SERS effect in the presence of Ag NPs on ULVs.

molecules on the surface of the ULVs which are in direct contact with Ag NPs.

In order to gain a better understanding of a hot-spot formation, we have monitored and analyzed time evolution of bright sites as shown in Figure 5. Time series of SERS spectra were recorded with an acquisition time of 0.4 seconds/spectrum and revealed fluctuating behaviors of some peak intensities and peak/peak intensity ratios. Raman bands exhibit dramatic intensity swings over time. While spectral positions of the bands are stable, some alterations in their intensities were observed.

Vibrational modes of $\mathrm{O}-\mathrm{C}-\mathrm{C}-\mathrm{N}^{+}$backbone of the DPPC molecule, in the range of $700-960 \mathrm{~cm}^{-1}$, show fluctuations. Both trans conformation vibration modes and gauche conformation vibration modes in the range of $720-870 \mathrm{~cm}^{-1}$ can be observed and peak/peak intensity ratios change within time. ${ }^{22}$ Other vibrational modes also show both gauche and trans conformational characteristics. Therefore, the vast variety of peaks exhibit swings in their intensities as can be seen in the Figure 5.

Even though a large number of molecules are averaged in order to record sample data, the resulting spectrum is dominated by spectral fluctuations of only a small number of molecules and the highest enhancements are attributed to Ag NPs loaded onto liposome surfaces. ${ }^{23,24}$ SERS enhancement factors ranging from $10^{6}$ to $10^{8}$ were estimated from these measurements. This is a very common level observed in many experimental measurements. ${ }^{25}$ Single molecule sensitivity was initially attributed to extremely high enhancement factors (from $10^{9}$ to $10^{12}$ ) but later claimed to be feasible under much lower enhancement (from $10^{6}$ to $10^{8}$ ). ${ }^{26}$

No SERS signal could be detected from the bare ULVs deposited onto the metallic Ag film, confirming the vital role of the Ag NPs loaded onto the ULVs to observe the SERS enhancement as well as the negligible role of the Ag metallic film used as the substrate.

Figure 6 displays the SERS spectra obtained from a potentially individual Ag NPs loaded ULV placed at the center of a hot-spot and those from darker sites around the hot spot. Travelling from brighter site to darker site, the intensities of SERS spectra decrease. All the raman bands were almost completely quenched at the darkest points where junctions of Ag NPs do not exist.

Due to the vast variety of functional groups in the DPPC molecule, a great number of prominent Raman bands could be observed. It is notable that bands attributable to the head group of the DPPC molecule were more prominent than those associated with tail groups, possibly due to the former's close proximity to Ag NPs leading to a local electrical field enhancement. Accordingly, the prominent raman modes at $700,960 \mathrm{~cm}^{-1}$ and at 1100 , $1225 \mathrm{~cm}^{-1}$ are assigned to $\mathrm{N}^{+}-\left(\mathrm{CH}_{3}\right)_{3}$ streching vibrations and $\left(\mathrm{PO}_{2}\right)^{-}$streching vibrations, respectively. Other skeletal Raman modes agree well with those reported in the literature for the DPPC moelcule. ${ }^{22,27,28}$ The Raman band intensities are almost 2 orders of magnitude higher in the hot-spot than in the spectrum collected outside this area. The resulting enhancement factor is about $10^{8}$.

For chemically specific imaging of biological samples, the Raman effect has been recently designated as a photostable mechanism; ${ }^{29}$ however, our results show that under intense illumination photostability may become a limiting issue in the SERS imaging.

Accordingly, the Ag NPs loaded ULVs eventually undergo carbonization during prolonged imaging under intense laser beams. When the carbonation takes place, the well-defined peaks disappear and the spectrum is dominated by only two broad bands that appear at 1380 and $1580 \mathrm{~cm}^{-1}$ as illustrated in Figure 7. The results are in good agrement within the literature $\mathrm{e}^{30-32}$ and demonstrate the possibility of laser induced photothermal therapy.

We observed a red-shift at the SPR band of Ag NPs after incorporating them with the ULVs. The position of the SPR band can be discussed within framework of Drude model. ${ }^{33}$ According to the Drude model, position of a SPR band of a spherical particle depends on the dielectric 


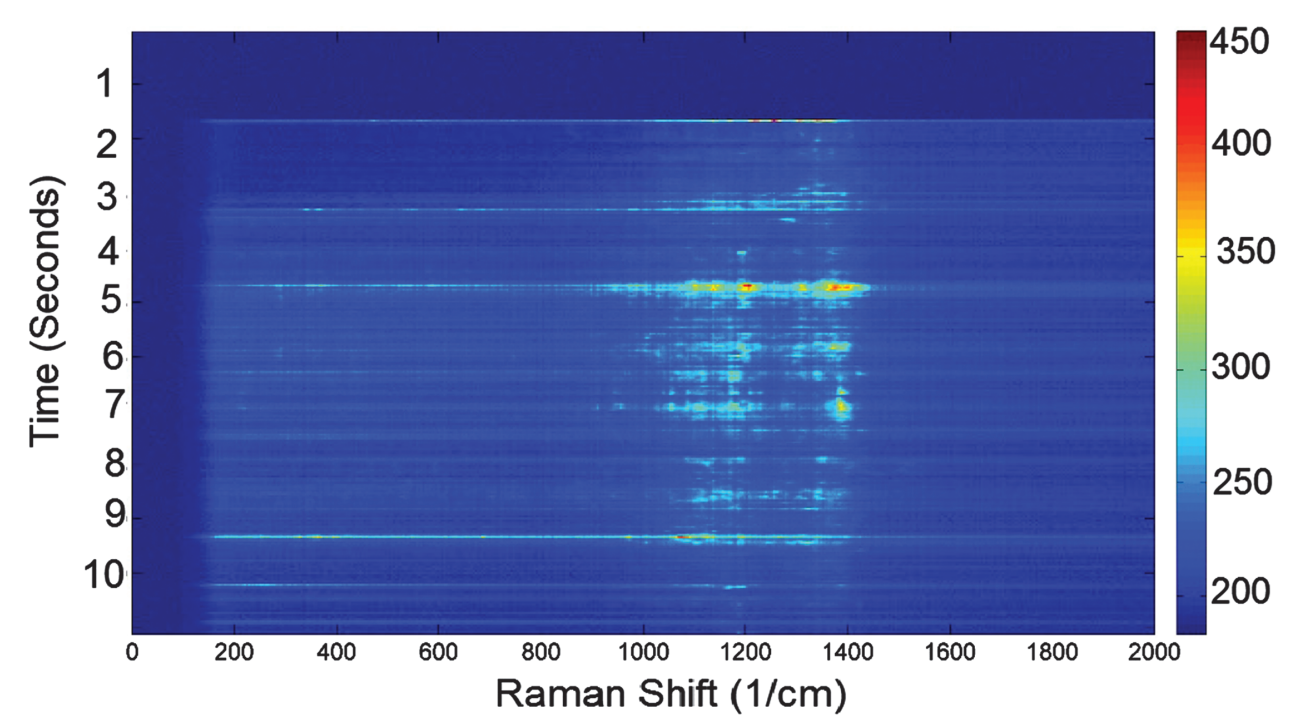

Figure 5. Waterfall plots of time series of SERS spectra of metallic Ag substrate which contains Ag NPs loaded ULVs.

constant of surrounding medium. The SPR band $(\lambda)$ of a spherical particle can be expressed as follows; ${ }^{34}$

$$
\lambda^{2}=\lambda_{p}^{2}\left(\varepsilon^{\infty}+2 \varepsilon_{m}\right)
$$

where $\lambda_{p}$ is the bulk plasma wavelength, $\varepsilon^{\infty}$ is the highfrequency dielectric constant due to interband and core transitions, and $\varepsilon_{m}$ is the dielectric constant of the surrounding medium. Therefore, the presence of a SPR band and the red shift in its magnitude imply the loading of the Ag NPs onto ULVs. In the case of dimers of spherical Ag NPs ( $\sim 25 \mathrm{~nm}$ radius), a shift of the resonance wavelength is expected as a function of the dimer separation $g$ as $; ;^{35}$

$$
\lambda_{\text {res }}=\lambda_{\text {inf }}\left(1+\left(g_{1} / g\right)^{0.6}\right)
$$

where $g_{1}=0.296 \mathrm{~nm}$, and $\lambda_{\text {inf }}=416 \mathrm{~nm}$ is the resonance wavelength at infinite separation. The maximum local field

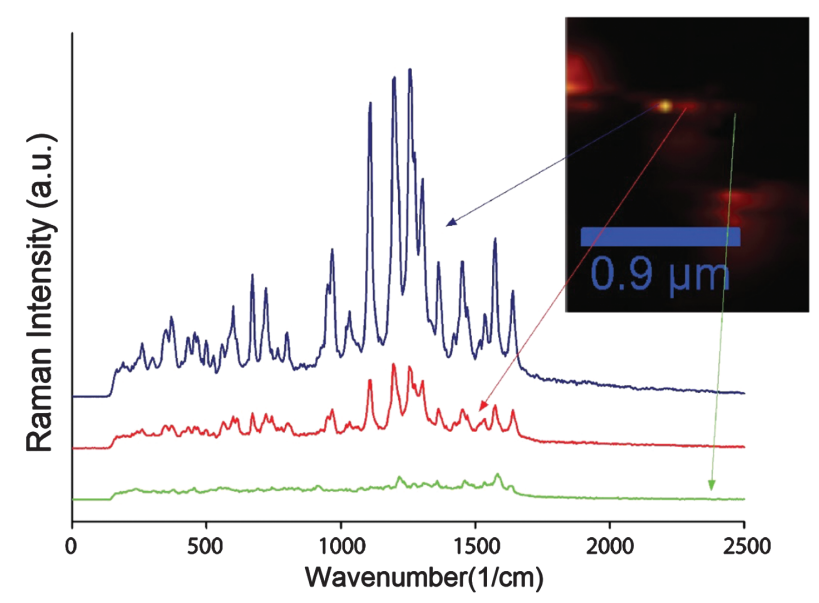

Figure 6. SERS spectra of DPPC constructing Ag NPs loaded ULVs from three specific points: at top of brighest point, so-called hot-spot (blue line), in between hot-spots (red line) and at darkest point (green line). enhancement $M_{\mathrm{LOC}}$ of the dimer is close to the point of minimum separation between the Ag NPs and is given similarly by

$$
M_{\mathrm{LOC}}=M_{\mathrm{inf}}\left(1+\left(g_{2} / g\right)^{2.5}\right)
$$

where $g_{2}=18 \mathrm{~nm}$, and $M_{\mathrm{inf}}=393$ is the field enhancement of individual NPs. Therefore, we would expect the field enhancement and resonance wavelength to fluctuate as a result of the Brownian motion of NPs forming the dimers on ULVs. This would cause the SERS signal intensity to also fluctuate in time.

Observed alterations in the intensities of the bands may be associated with three common mechanisms. Namely, diffusion of molecules, laser induced structural changes and Brownian motion can cause fluctuating behaviour in the SERS signals. ${ }^{36}$ The former one can be ruled out since

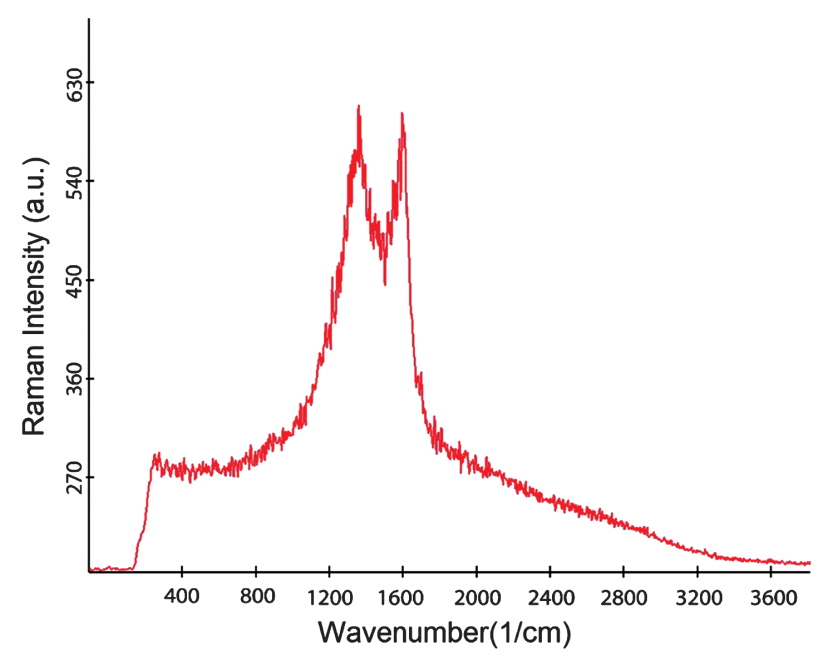

Figure 7. A broad double-peak between $1300 \mathrm{~cm}^{-1}$ to $1600 \mathrm{~cm}^{-1}$ due to the carbonization of the sample. 
it is impossible for such big ULVs to diffuse into homogeneous metallic Ag film substrate. Therefore, the fluctuations are mainly attributed to mechanisms related to laser induced structural changes and local Brownian motion of the individual Ag NPs loaded ULVs. The former can be attributed to conformational changes between gauche and trans conformations of the DPPC chains like a study of Chandra et al. ${ }^{37}$ where shifts of the Raman peak positions were attributed to conformational changes in lysozyme molecule adsorbed on Ag colloids.

Another common mechanism leading to the fluctuation of SERS signals is local Brownian motion of the $\mathrm{Ag}$ NPs loaded ULVs. The junctions between the Ag NPs are affected due to change in interconnections between the Ag NPs leading to the fluctuations. ${ }^{38}$ In the literature, similar fluctuating behaviors have been assigned to SERS signatures of single molecules. In the presence of strong enhanced local fields, those are rendered visible. ${ }^{36,38,39}$

\section{CONCLUSION}

In conclusion, constructed blinking hot-spots were observed on the surfaces of Ag NPs loaded ULVs and these hot-spots revealed higher Raman band intensities compared to the intensities detected from the darker sites. The blinking behaviour is attributed to possible laser induced conformational change of DPPC chain and local Brownian motion of liposomal spheres. Under prolonged laser exposure liposomes were carbonized demonstrating possibility of liposome usage in photothermal therapy by SERS tecnique.

Acknowledgment: PT would like to thank Tugba Endogan (Middle East Technical University, Central Laboratory) for assistance with the CTEM studies, Ege Ozgun (Bilkent University, Department of Physics) and Alper Devrim Özkan (Bilkent University, UNAM) for their helpful advices. IT thanks to Dr. Alper Kursat Ozturk for critically reading the manuscript.

\section{References and Notes}

1. M. Liu, L. Gan, L. Chen, Z. Xu, D. Zhu, Z. Hao, and L. Chen, J. Am. Chem. Soc. 28, 10725 (2012).

2. S. Li, B. Goins, L. Zhang, and A. Bao, Bioconjugate Chem. 23, 1322 (2012).

3. S. Ibsen, M. Benchimol, D. Simberg, C. Schutt, J. Steiner, and S. Esener, J. Control. Release 155, 358 (2011).

4. G. Wu, A. Mikhailovsky, H. A. Khant, C. Fu, W. Chiu, and J. A. Zasadzinski, J. Am. Chem. Soc. 130, 8175 (2008).

5. J. Kneipp, H. Kneipp, and K. Kneipp, Chem. Soc. Rev. 37, 1052 $\underline{(2008)}$
6. P. W. P. Nicholas and F. A. Ricardo, Chem. Soc. Rev. 37, 946 (2008).

7. I. R. Nabiev and H. Morjani, M. Eur. Biophys. J. 19, 311 (1991).

8. I. Chourpa, H. Morjani, and J. F. Riou, FEBS Lett. 397 (1996).

9. M. C. Daniel and D. Astruc, Chem. Rev. 104, 293 (2004).

10. C. Burda, X. Chen, R. Narayanan, and M. A. El-Sayed, Chem. Rev. 105,1025 (2005).

11. S. Link and M. A. El-Sayed, J. Phys. Chem. B 103, 8410 (1999).

12. X. Huang, P. K. Jain, I. H. El-Sayed, and M. A. El-Sayed, J. Lasers. Med. Sci. 23, 217 (2008).

13. P. K. Jain, I. H. El-Sayed, and M. A. El-Sayed, Nano Today 2, 18 (2007).

14. A. O. Govorov and H. H. Richardson, Nano Today 2, 30 (2007).

15. C. Kojima, Y. Hirano, E. Yuba, A. Harada, and K. Kono, Colloids Surf. B 66, 246 (2008)

16. C. Chen, S. Zhu, T. Huang, S. Wang, and X. Yan, Anal. Methods 5, 2150 (2013)

17. N. C. M. Tam, B. M. T. Scott, D. Voicu, B. C. Wilson, and G. Zheng, Bioconjugate Chem. 21, 2178 (2010).

18. X. Liu, S. Huan, Y. Bu, G. Shen, and R. Yu, Talanta 75, 797 (2008).

19. H. W. Cheng, S. Y. Huan, H. L. Wu, G. L. Shen, and R. Q. Yu, Anal. Chem. 81, 9902 (2009).

20. H. Xu, E. J. Bjerneld, M. Kall, and L. Borjession, Phys. Rev. Lett. 83, 4357 (1999).

21. M. Kahraman, A. I. Zamaleeva, R. F. Fakhrullin, and M. Culha, Anal. Bioanal. Chem. 395, 2559 (2009).

22. M. Harrand, Chem. J. Phys. 81, 1 (1984).

23. Y. Sawai, B. Takimoto, H. Nabika, K. Ajito, and K. Murakoshi, J. Am. Chem. Soc. 129, 1658 (2007).

24. D. R. Ward, N. K. Grady, C. S. Levin, N. J. Halas, and Y. Wu, Nano Lett. 7, 1396 (2007).

25. H. Ko, S. Singamaneni, and V. V. Tsukruk, Small 4, 1576 (2008).

26. E. C. Le Ru and P. G. Etchegoin, Annu. Rev. Phys. Chem. 63, 65 (2012).

27. Y. Xu, H. Yang, Y. Yan, and Z. Zhang, J. Photochem. Photobiol B: Biol. 45, 179 (1998).

28. Z. D. Schultz and I. W Levin, Annu. Rev. Anal. Chem. 4, 343 (2011).

29. J. W. Kang, F. T. Nguyen, N. Lue, R. R. Dasari, and D. A. Heller, Nano Lett. 12, 6170 (2012).

30. X. Yiming, Z. Zhixiang, Y. Hongying, X. Yan, and Z. Zhiyi, J. Photochem. Photobiol B: Biol. 52, 30 (1999).

31. A. Kudelski and B. Pettinger, Chem. Phys. Lett. 321, 352 (2000)

32. D. Pradhan and M.Sharon, Electrochim. Acta 50, 2905 (2005).

33. P. Drude, Ann. Phys. 306, 566 (1900).

34. P. Mulvaney, Langmuir 12, 788 (1996).

35. E. C. Le Ru and P. G. Etchegoin, Principles of Surface-Enhanced Raman Spectroscopy and Related Plasmonic Effects, 2nd edition, Elsevier, Amsterdam (2009).

36. E. C. Le Ru and P. G. Etchegoin, Principles of Surface-Enhanced Raman Spectroscopy and Related Plasmonic Effects, 1nd edition, Elsevier, Amsterdam (2008).

37. G. Chandra, K. S. Ghosh, S. Dasgupta, and A. Roy, Int. J. Biol. Macromol. 47, 361 (2010).

38. S. Ayas, G. Cinar, A. D. Ozkan, Z. Soran, O. Ekiz, D. Kocaay, A. Tomak, P. Toren, Y. Kaya, I. Tunc, H. Zareie, T. Tekinay, A. B. Tekinay, M. O. Guler, and A. Dana, Scie. Rep. 3, 2624 (2013).

39. K. A. Bosnick, J. Jiang, and L. E. Brus, J. Phys. Chem. B 106, 8096 $\underline{(2002)}$

Received: 19 June 2016. Accepted: 8 September 2016. 